\title{
Integration and testing of the Mini-EUSO telescope
}

\author{
G. Cambiè ${ }^{1}$ and L. Marcelli ${ }^{1}$ \\ for the JEM-EUSO collaboration \\ e-mail: giorgio.cambie@roma2.infn.it \\ ${ }^{1}$ INFN and University of Rome Tor Vergata, Physics Department, via della Ricerca Scientifica 1, 00133 , \\ Roma (RM).
}

\begin{abstract}
The Mini-EUSO telescope, approved by ASI (Italian Space Agency) and Roscosmos (Russian Space Agency) as long-term program of space experiments to be launched on the Progress spacecraft during the Expedition 60/61 in 2019, will be placed on a nadir-facing, UV transparent window on the ISS Russian Zvezda module looking down on Earth. As one of the JEM-EUSO collaboration missions, its first objective is to observe UV light produced by UHECR (Ultra High Energy Cosmic Ray) events from upper atmosphere ( $\sim 400 \mathrm{~km}$ altitude), moreover, from this point of view, Mini-EUSO will perform, for the first time, high-resolution mapping of night-earth UV (300 - 400 $\mathrm{nm}$ ) emission, focusing on terrestrial background sources, TLEs (Transient Luminous Events), bioluminescence, meteoroids burning in atmosphere, search for SQM (Strange Quark Matter) signals, as well as detecting and mapping space debris. The integration of the instrument, currently underway at the University of Rome Tor Vergata, is at an advanced stage in order to be compliant with a launch opportunity in June 2019. The on ground test results will be presented.
\end{abstract}

\section{Introduction}

Along with TA-EUSO, EUSO-Balloon and EUSO-SPB, Mini-EUSO belongs to the JEM-EUSO collaboration missions [1], which involves more than 16 countries, 93 institutes and 351 people all over the world. The JEM-EUSO collaboration aims to detect UV light produced by UHECR-induced extensive air showers (EAS) due to the interaction in atmosphere with $N_{2}$ molecules from the vantage point of low Earth orbit, thereby largely increasing the effective detector volume [2]. Mini-EUSO instrument comprises a compact telescope of dimensions $37 \times 37 \times 62 \mathrm{~cm}^{3}$ with a large field of view $\left( \pm 22^{\circ}\right)$, based on an optical system employing a focusing optics, a sensor array of MAPMTs (Multi Anode PhotoMultiplier Tubes) with its read-out electronics and different ancillary sensors for complementary measurements in the near infrared (NIR) $(1500-1600 \mathrm{~nm})$ and visible (VIS) (400 - 780 $\mathrm{nm}$ ) range.

\section{Instrument overview}

Mini-EUSO instrument comprises two double sided PMMA Fresnel lenses, $25 \mathrm{~cm}$ in diameter, which will focus light onto a focal surface, called PDM (Photo Detector Module), consisting of an array 
of 36 Hamamatsu Photonics (model R11265-M64) MAPMTs. Details are shown in table 2a. Each dinodes of MAPMTs is powered by a Cockroft-Walton high voltage power supply placed inside a ceramic pad. All MAPMTs are covered by a $2 \mathrm{~mm}$, anti-reflective, BG3 UV filter placed on the entry window. In addition to the main detector sensible in the UV range, Mini-EUSO contains two ancillary cameras, NIR and VIS, provided by PointGrey. All subsystems are shown in fig. 1.

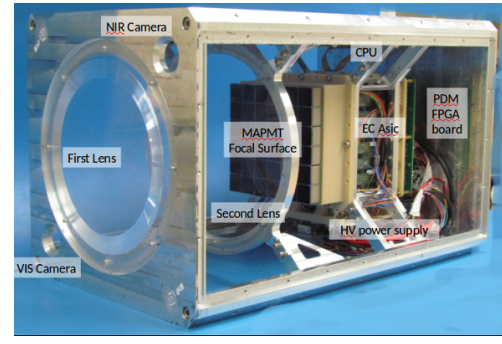

(a) Mini-EUSO telescope body

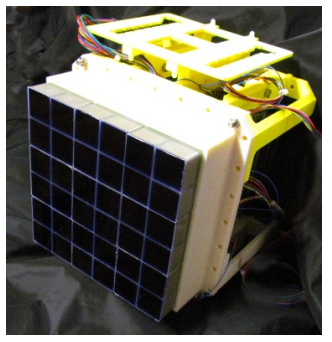

(b) Mini-EUSO PDM

Figure 1: Mini-EUSO apparatus

Furthermore, for the first time in space, Mini-EUSO telescope will take advantage of taking data through a $8 \times 8$ channels SiPM-MPPC (Silicon PhotoMultiplier-Multi Pixel Proportional Counter) C14047 Hamamatsu array. The data processing chain comprises three dedicated SPACIROC3 Asic boards, a Xilinx Zynq XC7Z030 system on chip (Kintex7 FPGA), a PCIe/104 form factor CPU and an ATMegaS128 rad hard based board for analog sensors read out with a sampling rate of $2.5 \mu \mathrm{s}$ called Gate Time Units (GTUs). The LVPS (Low Voltage Power Supply) feed the entire Mini-EUSO subsystem which filters and stabilize $27 \mathrm{~V}$ coming from ISS, thus preserving the entire instrumentation from spike or polarization inversion through several Vicor DC/DC Converters. A scheme of power supply is shown in fig. 2 b.

\begin{tabular}{lr}
\hline Lens Dimension & $25 \times 11 \mathrm{~mm}^{2}$ \\
Focal Lenght & $30 \mathrm{~cm}$ \\
$\mathrm{~N}^{\circ}$ of Pixels & 2304 \\
FoV $/$ PDM & $44 / 2.6 \times 10^{2} \mathrm{~km}$ \\
FoV $/$ pixel & $0.8 / 6.11 \mathrm{~km}$ \\
Mass & $\sim 27 \mathrm{~kg}$ \\
Power Consumption & $\leq 60$ \\
\hline
\end{tabular}

(a) Mini-EUSO key parameters

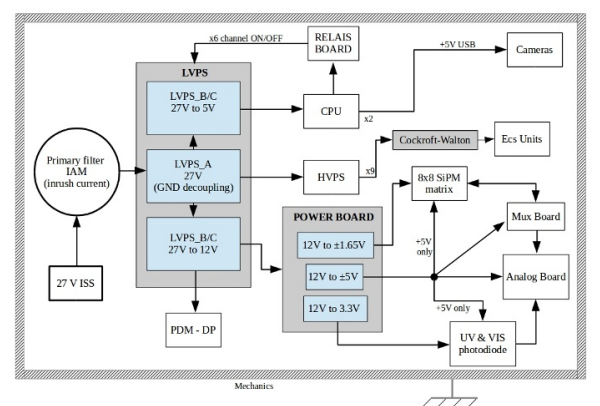

(b) Mini-EUSO subsystem power distribution scheme

Figure 2 


\section{Integration and test}

The JEM-EUSO collaboration worked on the integration of the apparatus during whole 2017 and 2018 focusing on: mechanics and supports assembly, operational tests on electronic boards, subsystem interfaces, instrumentation response and efficiency. In fig. 3 are shown some of the apparatus integration basic steps and the test bench setup done at the hosting institutes: Universite Paris Diderot - APC laboratoire (Astroparticule et Cosmologie), Riken research institute (Wako, Tokyo) and University of Rome Tor Vergata. We focused our attenction on the qualification and efficiency test on the Photo Detector Module comprising the MAPMTs array and the front-end electronics. Several aspects have been taken into account.

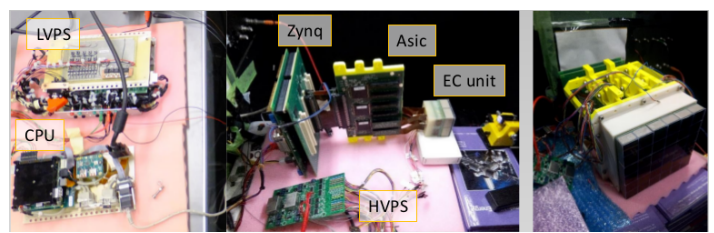

(a) Test bench inside a black box for electronics operational tests and MAPMTs response

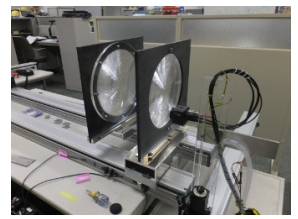

(b) Test on fresnel lenses point spread function through a laser shot

Figure 3: Mini-EUSO Integration and test bench procedure

Tests on the correct operativity, power supply and response of the whole detector were made, as well as the PMTs efficiency analysis to provide the correct single photoelectron counting mode working point, through Asic ADC graphical analysis response (S-curve). This can be achieved from fig. 5b, where in the top we have a sample of S-curve which shows counts as a function of ADC channels while on bottom its slope analysis allows to determine the right ADC channel working point. Simulation of PDM response to the signal produced by interested events such as UHECR (fig. 5c), meteoroids and SQM efficiency detection by magnitude (fig. 5d), satellite and space debris passage on the field of view, star classification and so on [4] were made. Most of the work was done through the ETOS (Euso TO Screen) scientific software which was expressly generated for all experiments belonging to the JEM-EUSO program. It is the main quick-view and quick-analysis software written both in Python and $\mathrm{C}++$ which operates on the ROOT files generated with ETOT (Euso TO Tree) from the raw CPU data. A demonstration on how ETOS works is shown in fig. 4.

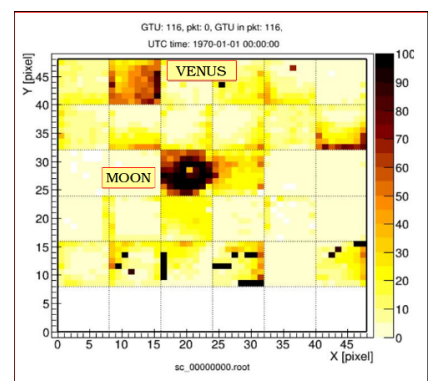

Figure 4: Tokyo night time PDM reconstruction with ETOS software. 


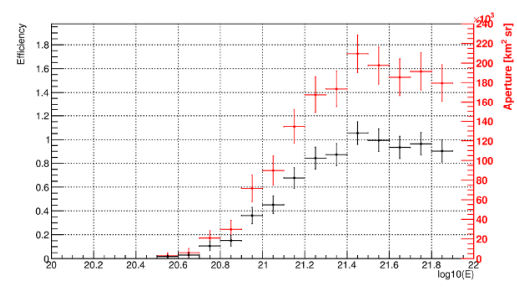

(a) The detection efficiency (on the left axis, in black) and geometric aperture (on the right axis, in red) as a function of the EAS energy in $\mathrm{eV}$ [3]
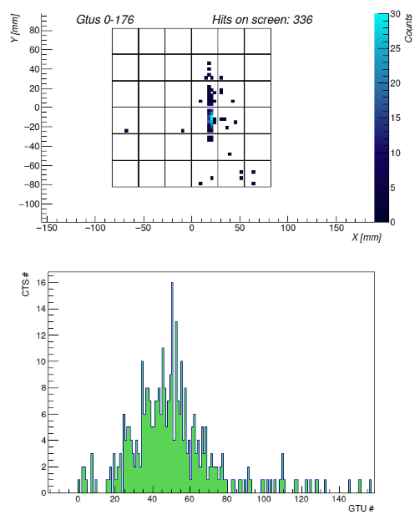

(c) Top: Photon counts observed in the Mini-EUSO focal surface for a simulation of an $E=1 \times 10^{21} \mathrm{eV}$ event with an inclination of $80^{\circ}$ to the nadir. Bottom: Light curve for the same event [4]

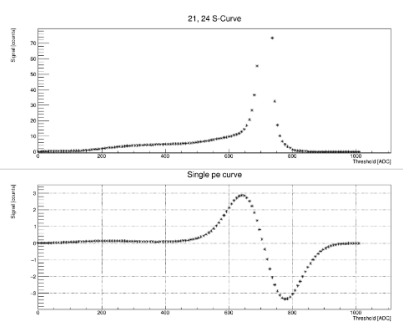

(b) Single pixel S-curve and its derivative in order to understand the single photoelectron work point
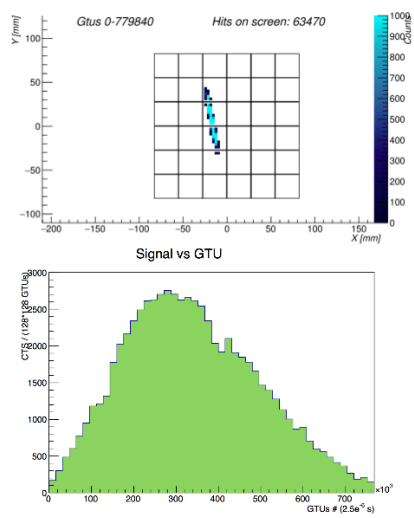

(d) Top: Expected light track of a meteor of absolute magnitude $\mathrm{M}=+5$. Bottom: Expected light profile. Each time bin on $\mathrm{x}$-axis corresponds to an integration time of $40.96 \mathrm{~ms}$, corresponding to Mini-EUSO data trigger level 3 [4]

Figure 5: Mini-EUSO PDM efficiency analysis and simulations

\section{References}

[1] M. Casolino, A. Belov, M. Bertaina, G. Cambie', F. Capel, T. Ebisuzaki, P. Klimov, M.I. Panasyuk, P. Picozza, M. Ricci, Proceedings of Science, 301, 369 (2017). PoS(ICRC2017)369

[2] A. V. Olinto, E. Parizot, M. Bertaina, and G. Medina-Tanco, Proceedings of the 34th International Cosmic Ray Conference 236, (2015), PoS(ICRC2015)623,

[3] F. Capel, A. Belov, M. Casolino, P. Klimov, Advances in Space Research 62(10), 2954-2965, (2018), 1729.00405.

[4] M. Bertaina, S. Biktemerova, K. Bittermann, et al., Advances in Space Research, 53(10), 15151535, (2014). 